\title{
Lower urinary changes over time in suprasacral spinal cord injury
}

\author{
DD Cardenas ${ }^{1}$, ME Mayo ${ }^{3}$ and LR Turner ${ }^{2}$ \\ ${ }^{1}$ Professor, ${ }^{2}$ Research Assistant, Department of Rehabilitation Medicine, RJ-30; ${ }^{3}$ Professor, Department of \\ Urology, RL-10, University of Washington, Seattle, WA 98195, USA
}

This paper reviews 179 patients (23 females and 156 males) with suprasacral spinal cord injury (SCI) who underwent videourodynamic evaluation to compare maximum detrusor pressure, compliance, and trabeculation with methods of bladder management, years postinjury, and age. The patients were divided into four groups based on mode of bladder management: clean intermittent catheterization (CIC), indwelling catheter (IND), external collector (EC), and voiding (V). Maximum pressure decreased significantly with increasing age for those using EC $(P<0.01)$ and CIC $(P<0.05)$. Maximum pressure also decreased significantly with years post-injury for patients on EC $(P<0.01)$ and was highest the first decade after injury and progressively decreased through the fifth decade. Post-hoc tests indicated more severe trabeculation in patients in the EC group than in either the CIC or IND groups. Age and trabeculation did not correlate in those on EC. We conclude that patients with long-standing suprasacral SCI using EC are more likely to have lower detrusor pressures than are those with less chronic SCI. This finding may reflect the effects of age as well as reduced survival in those using EC with chronically elevated detrusor pressure.

Keywords: spinal cord injury; urinary tract; ageing

\section{Introduction}

The number of older persons with spinal cord injury (SCI) is increasing and has led to a growing interest in aging research. ${ }^{1-4}$ However, studies of the neuropathic bladder have been primarily conducted in younger persons. Recently Perkash (1993) reviewed many potential problems of the elderly patient with SCI that may affect the bladder. ${ }^{5}$ These include benign prostatic hyperplasia, cancer of the prostate and bladder, other neurological disorders, diabetes mellitus, obesity, and congestive heart failure. These conditions can render the current bladder management inappropriate. In addition to medical conditions per se, medications used to treat certain conditions also affect urinary output and function. Only a few studies have addressed the older person with SCI, or those aging with SCI. ${ }^{6-8}$ Madersbacher and Oberwalder (1987) studied 26 subjects who had a spinal cord injury after the age of 60 and concluded that age makes bladder rehabilitation more difficult because of the decreased ability of the elderly to cope with the new situation, because of pre-existing conditions such as prostatic enlargement in men and cystocele in women, and because of a delayed and sometimes weak detrusor reflex. ${ }^{8}$ They also studied 29 subjects over the age of 60 with a spinal cord injury for more than 20 years and found a high percentage of secondary structural changes due to persisting functional outflow obstruction. However, no urodynamic data were described. The purpose of this study was to determine what urodynamic changes occur in the lower urinary tract in relationship to age, duration of injury and bladder management in patients with SCI.

\section{Method}

Subjects

Subjects were 179 patients ( 23 female, 156 male) with suprasacral SCI. Twelve patients had high tetraplegia (C1-4); 100 patients had levels of C5-8; and 67 patients had levels of T1-L2. The patients were divided into four groups based on the predominant mode of bladder management just prior to or at the time of study: clean intermittent catheterization (CIC), indwelling catheter (IND), external collector (EC), and voiding without using any form of catheter (V). The CIC group consisted of 74 patients with a mean age of 34 years (range 16-70); the IND group consisted of 30 patients with a mean age of 41 years (range 19-77); the EC group consisted of 62 patients with a mean age of 37 years (range 18-79); and the $\mathrm{V}$ group consisted of 13 patients with a mean age of 36 years (range 18-56). With regard to completeness of the lesions, $89 \%$ of the CIC group, $77 \%$ of the IND group, $86 \%$ of the EC group, and $46 \%$ of the $\mathrm{V}$ group were complete. The remainder in each group were incomplete. The mean years post-injury for patients in each group is shown in Table 1. 
Table 1 Mean number of years post-injury

\begin{tabular}{llr}
\hline Group & $n$ & Mean $(S D)$ \\
\hline CIC & 74 & $3.8(6.7)$ \\
IND & 30 & $12.4(8.7)$ \\
EC & 62 & $10.7(9.4)$ \\
V & 13 & $9.2(8.5)$ \\
\hline
\end{tabular}

\section{Procedure}

Patients underwent videourodynamic evaluation consisting of simultaneous intravesical pressure, abdominal pressure and needle or surface electromyography. The bladder pressure was measured with a $7 \mathrm{~F}$ double lumen catheter using liquid contrast at a filling rate of $50 \mathrm{ml} \mathrm{min}^{-1}$. Abdominal pressure was measured with a small feeding tube. Intermittent fluoroscopy was performed with a recording of the study on videotape. Reasons for urodynamic evaluation were routine evaluation, recurrent septic episodes, autonomic dysreflexia, failure to empty adequately, and incontinence. Some patients with SCI for 20 years or more were recruited for urodynamics as part of a long-term study. Patients were requested to discontinue any anticholinergic medication at least $72 \mathrm{~h}$ prior to study, and none had symptomatic urinary tract infection at the time of the study.

\section{Definitions}

Maximum pressure was defined as the peak detrusor pressure (bladder pressure minus abdominal pressure) in $\mathrm{cm} \mathrm{H}_{2} \mathrm{O}$ recorded during urodynamics, usually associated with a bladder contraction. If no contraction was present, the baseline pressure rise due to bladder compliance gave maximum pressure. Capacity was defined as the maximum volume reached before a detrusor contraction, leakage of urine, a sensation of fullness, autonomic dysreflexia, or $600 \mathrm{cc}$ if none of the former events occurred. Compliance was defined as the ratio of the change in bladder volume to the associated change in baseline pressure determined at capacity. If a bladder contraction occurred, the pressure recorded just prior to the contraction was used. Trabeculation was clinically graded on a 4 point scale with 0 indicating no trabeculation, 1 indicating mild trabeculation, 2 indicating moderate trabeculation, and 3 indicating severe trabeculation.

\section{Data analysis}

Analysis of variance (ANOVA) was used to compare maximum pressure, compliance, and trabeculation across method of bladder management, years postinjury, and age: Additionally, a Pearson correlation matrix was computed for maximum pressure, trabeculation, compliance, years post-injury, and age.

\section{Results}

\section{Maximum pressure}

Table 2 presents mean maximum pressures in $\mathrm{cm}_{2} \mathrm{O}$ for each bladder management group. ANOVA re-
Table 2 Mean maximum pressures $\left(\mathrm{cm} \mathrm{H}_{2} \mathrm{O}\right)$

\begin{tabular}{lll}
\hline Group & $n$ & Mean $(S D)$ \\
\hline CIC & 74 & $65.0(48.5)$ \\
IND & 30 & $42.3(25.4)$ \\
EC & 62 & $70.8(44.0)$ \\
V & 13 & $81.5(38.0)$ \\
\hline
\end{tabular}

vealed significant differences between management groups $[\mathrm{F}(3,175)=3.76, P<0.01]$. A post-hoc Tukey HSD multiple comparison indicated that maximum pressure for those using IND was significantly less than for those using EC or V. Maximum pressure decreased significantly with increasing age for those using EC $(r=-0.32, P<0.01)$ and CIC $(r=-0.24, P<0.05)$. Maximum pressure decreased significantly with years post-injury for patients on EC $(r=-0.40, P<0.01)$ (Figure 1), but not for patients on CIC, IND, or V $(P>0.05)$. For those on EC, maximum pressure was highest the first decade after injury and progressively decreased through the fifth decade (see Figure 1).

\section{Capacity}

Table 3 presents mean capacity $(\mathrm{ml})$ for each management group. ANOVA revealed significant differences between management groups $[\mathrm{F}(3,175)=11.037$, $P<0.001$ ]. Patients in both the CIC and EC groups had significantly higher capacity than patients in the IND group.

\section{Compliance}

Table 4 presents mean compliance for each management group. The mean compliance of patients on IND was abnormally low $(18.28, \mathrm{SD}=22.38)$, while compliance of patients on CIC was the highest (41.92, $\mathrm{SD}=27.95)$. ANOVA revealed significant differences

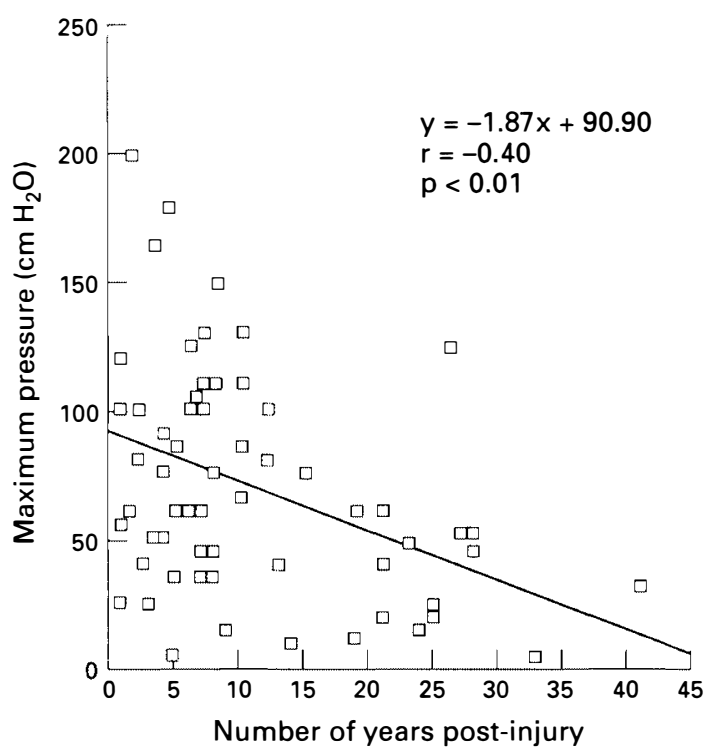

Figure 1 Years post-injury $v s$ maximum pressure for patients using EC 
Table 3 Mean capacity (ml)

\begin{tabular}{lll}
\hline Group & $n$ & Mean $(S D)$ \\
\hline CIC & 74 & $303.0(164.0)$ \\
IND & 30 & $128.8(117.7)$ \\
EC & 62 & $250.4(129.9)$ \\
V & 13 & $213.1(102.3)$ \\
\hline
\end{tabular}

Table 4 Mean compliance

\begin{tabular}{lll}
\hline Group & $n$ & Mean $(S D)$ \\
\hline CIC & 74 & $41.9(28.0)$ \\
IND & 30 & $18.3(22.4)$ \\
EC & 62 & $35.5(26.7)$ \\
V & 13 & $31.5(13.9)$ \\
\hline
\end{tabular}

between management groups $[\mathrm{F}(3,175)=6.02, P<$ 0.001]. Patients in both the CIC and EC groups had significantly higher compliance than patients in the IND group. However, compliance of patients on CIC was not significantly different than compliance of those on $\mathrm{EC}(P<0.05)$.

\section{Trabeculation}

Figure 2 presents a graphical comparison of frequency of each grade of trabeculation for four management groups. ANOVA revealed significant differences in severity of trabeculation between management groups $[\mathrm{F}(3,175)=3.92, P<0.01]$. Post-hoc tests indicated more severe trabeculation in patients in the EC group than in either the CIC or the IND groups. Age and trabeculation were significantly correlated for patients on IND $(r=0.42, P<0.05)$, but not for patients on $\mathrm{CIC}$ or EC. The correlation between years post-injury

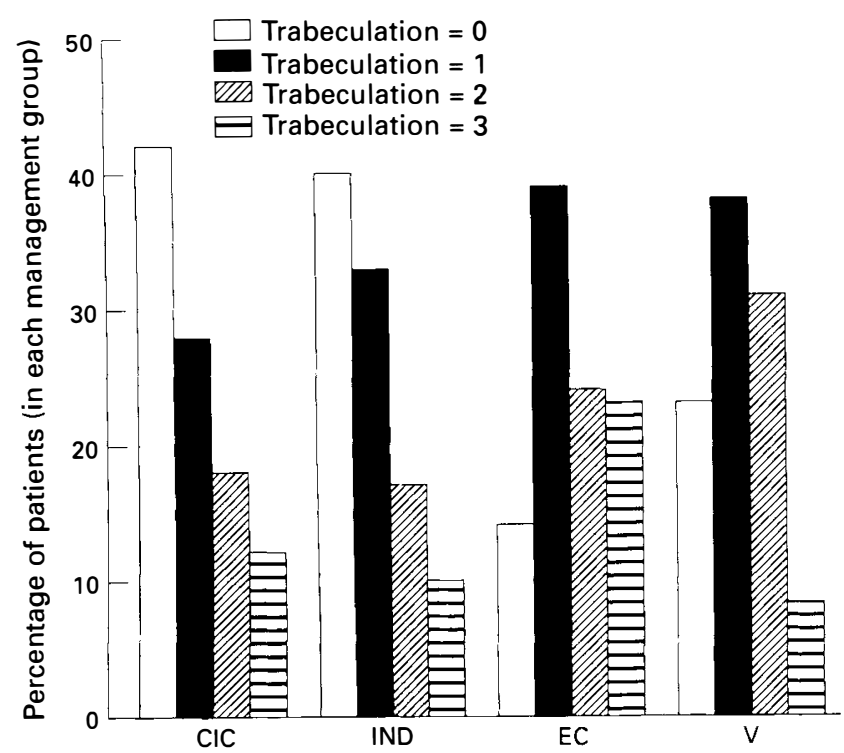

Figure 2 Percentage of patients with each degree of trabeculation, divided by management group and trabeculation was significant only for patients on $\mathrm{CIC}(r=0.29, P<0.05)$.

\section{Discussion}

Although it would have been ideal to compare the age changes in the SCI population with age and sex-matched cohorts, this would be a very extensive prospective study considering the size of our patient group. We did, however, compare our data with age changes in symptomatic and asymptomatic groups reported in the literature. Age-related changes in the normal bladder have been described with detrusor hyperactivity with impaired contractile function, the second most common cause of incontinence in 30 unselected nursing home patients, the majority of whom were women. ${ }^{9}$ Detrusor instability and urge incontinence in the male geriatric population may be an age-associated phenomenon and not secondary to obstruction. ${ }^{10}$ Clinically, in the non-SCI population frequency, urgency, and incontinence are common in the elderly as are poorly sustained contractions resulting in incomplete emptying with voiding. Some women may have incontinence related to impaired sphincter activity or stress incontinence.

There appears to be little information on normal bladder pressures in otherwise asymptomatic elderly males except a study by Spangberg et al (1990) ${ }^{11}$ and Andersen (1978). ${ }^{12}$ Spangberg et al (1990) found a median maximum detrusor pressure of $49 \mathrm{~cm} \mathrm{H}_{2} \mathrm{O}$ with a range of $16-139 \mathrm{~cm} \mathrm{H}_{2} \mathrm{O}$ and Andersen et al (1978) found mean maximum voiding detrusor pressures of $121 \mathrm{~cm} \mathrm{H} \mathrm{H}_{2} \mathrm{O}$ in elderly male volunteers who claimed to have normal voiding patterns. ${ }^{11,12}$ In the SCI population, studies generally combine age groups, duration of spinal cord injury, and method of bladder management, but in one study of chronic SCI, 20 patients at least 20 years post-injury underwent urodynamic evaluation and showed a mean total bladder capacity of $350 \mathrm{cc}$, with $55 \mathrm{~cm} \mathrm{H}_{2} \mathrm{O}$ mean maximum voiding pressure (range $30-120 \mathrm{~cm} \mathrm{H}_{2} \mathrm{O}$ ). ${ }^{6}$ Thus, from the few studies in asymptomatic elderly males maximum detrusor pressures appear to be higher than in elderly SCI patients.

The results of experimental studies on the aging bladder have been inconsistent. Some have suggested that age changes are related primarily to changes in neuronal innervation and central control of micturition rather than alterations in bladder contractility. ${ }^{13}$ Other authors have shown changes in cholinergic receptor density on the bladder base of a rat's bladder. ${ }^{14}$ The patients in this study were not studied on anticholinergics except in a few cases and thus represented the effects of a neurologic lesion above the sacral cord as well as age and duration of injury. The effect of any changes in cognitive function which may have affected bladder function or the patient's ability to perform a specific bladder program were not determined in this study.

This study examined the changes in the lower tract in subjects with suprasacral SCI and revealed some 
interesting findings: (1) older patients on CIC and EC had lower maximum bladder pressures than younger patients; (2) maximum pressure decreased with years post-injury for those on EC; (3) age and trabeculation did not correlate in those on EC; and (4) years post-injury correlated with severity of trabeculation in those on CIC, although age did not. The reason for the finding that older patients had lower bladder pressure may be that older patients are more likely to comply with chronic anticholinergic medication which could lead to greater bladder capacity in those on CIC, and older patients on an external collector may have better acceptance of treatment for outflow obstruction. Another possible reason is, of course, the natural aging processes that may lessen the overall strength of the detrusor contraction. This relative hypocontractility has been described in elderly patients with incontinence and incomplete emptying, but, on the contrary, asymptomatic elderly males have increased voiding detrusor pressure..$^{9,12}$

The finding of more severe trabeculation in those on EC is what one might expect; however, age was not correlated to trabeculation for those on EC and thus does not appear to be the reason for this finding. In a prior study, we reported a trend of increased trabeculation with age. ${ }^{15}$ However, the increasing age of those who incur a spinal injury may be changing this relationship.

Although we are not advocating indwelling catheter management in SCI patients, maximum pressure and trabeculation were less with this method of management and although capacity and compliance were worse, these may have been reversible changes secondary to the collapsed bladder status. Also upper tract changes on IND do not seem to be as prevalent as in the past so perhaps a well managed IND is preferable to a poorly managed EC or CIC program. ${ }^{6,7}$

Perhaps the most important finding of this study is that patients with long-term suprasacral SCI using an external collector were more likely to have lower bladder pressures than those with less chronic SCI. Whether those with persistent high pressures fail to survive as long as those with lower pressure can not be determined by the cross-sectional nature of our study, but may account in part for this finding as may the effects of age. The interplay of the spinal cord lesion, age, and other associated conditions with method of bladder management poses a complex problem that warrants further research.

\section{References}

1 Gerhart KA et al. Long-term spinal cord injury: functional changes over time. Arch Phys Med Rehabil 1993; 74: 1030-1034.

2 Eisenberg MG, Tierney DO. Changing demographic profile of the spinal cord injury population: implications for health care support systems. Paraplegia 1985; 23: 335-343.

3 Samsa GP, Patrick CH, Feussner JR. Long-term survival of veterans with traumatic spinal cord injury. Arch Neurol 1993; 50: $909-914$.

4 Menter RR. Spinal cord injury and aging: exploring the unknown. J Am Paraplegia Soc 1993; 16: 179-189.

5 Perkash I. Incontinence in patients with spinal cord injuries. Geriatr Urol 1994; 26: 321-325.

6 Chao R, Clowers D, Mayo ME. Fate of upper urinary tracts in patients with indwelling catheters after spinal cord injury. Urology 1993; 42: 259-262.

7 Dewire DM et al. A comparison of the urological complications associated with long-term management of quadriplegics with and without chronic indwelling urinary catheters. J Urol 1992; 147: 1069-1072.

8 Madersbacher G, Oberwalder M. The elderly para- and tetraplegic: special aspects of the urological care. Paraplegia 1987; 25: 318-323.

9 Resnick NM, Yalla SV. Detrusor hyperactivity with impaired contractile function. JAMA 1987; 257: 3076-3081.

10 Gormley EA et al. Effect of transurethral resection of the prostate on detrusor instability and urge incontinence in elderly males. Neurourol Urodynam 1993; 12: 445-453.

11 Spangberg A, Terio H, Ask P. Pressure/flow studies in elderly men without voiding problems: estimation of the urethral pressure/flow relation and urethral elasticity. Neurourol Urodynam 1990; 9: 123-138.

12 Andersen JT, Jacobsen O, Worm-Pedersen J, Hald T. Bladder function in healthy elderly males. Scand J Urol Nephrol 1978; 12: $123-127$.

13 Chun $\mathrm{Al}$ et al. Effects of age on urinary bladder function in the male rat. J Urol 1988; 141: 170-315.

14 Hayes EE, McConnell JA, Benson GS. The effect of aging on cholinergic receptor binding in the rat urinary bladder. Neurourol Urodynam 1983; 2: 311-315.

15 Hoffberg HJ, Cardenas DD. Bladder trabeculation and its associated urologic findings in the neurogenic bladder following spinal cord injury. Arch Phys Med Rehabil 1986; 67: 750-753. 